\title{
Low dose amiodarone and sotalol in the treatment of recurrent, symptomatic atrial fibrillation: a comparative, placebo controlled study
} G E Kochiadakis, N E Igoumenidis, M E Marketou, M D Kaleboubas, E N Simantirakis,
P E Vardas

\begin{abstract}
Objective-To assess and compare the safety and efficacy of amiodarone and sotalol in the treatment of patients with recurrent symptomatic atrial fibrillation.

Design-Prospective, randomised, single blind, placebo controlled study.

Setting-Tertiary cardiac referral centre.

Patients-186 consecutive patients (97 men, 89 women; mean (SD) age, 63 (10) years) with recurrent, symptomatic atrial fibrillation.

Interventions-65 patients were randomised to amiodarone, 61 to sotalol, and 60 to placebo. Patients receiving amiodarone were maintained at a dose of $200 \mathrm{mg} /$ day after a 30 day loading phase. The sotalol dose was $160-480 \mathrm{mg}$ daily, as tolerated.

Main outcome measures-Recurrence of atrial fibrillation or side effects.

Results-In the amiodarone group, 31 of the 65 patients developed atrial fibrillation after an average of six months, while 15 (11 in sinus rhythm and four in atrial fibrillation) experienced significant side effects after an average of 16 months. In the sotalol group, relapse to atrial fibrillation occurred in 47 of the 61 patients after an average of eight months; three experienced side effects during the titration phase. In the placebo group, 53 of the 60 patients developed atrial fibrillation after an average of four months $(p<0.001$ for amiodarone and sotalol $v$ placebo; $\mathrm{p}<0.001$ for amiodarone $v$ sotalol).

Conclusions-Both amiodarone and sotalol can be used for the maintenance of normal sinus rhythm in patients with symptomatic atrial fibrillation. Amiodarone is more effective but causes more side effects.
\end{abstract}

(Heart 2000;84:251-257)

Keywords: amiodarone; sotalol; atrial fibrillation

Various antiarrhythmic drugs, especially those belonging to class I (quinidine, procainamide, flecainide, propafenone), have been used to prevent recurrence of atrial fibrillation in patients with paroxysmal atrial fibrillation or with chronic atrial fibrillation after successful cardioversion. However, their use is fraught with problems, including incomplete efficacy, proarrhythmic properties, and possibly increased mortality. ${ }^{1-10}$

Recently, the development of new antiarrhythmic agents has focused on the class III mode of action - that is, the prolongation of myocardial repolarisation and refractoriness. $^{2-511-13}$ Only two antiarrhythmic agents that are available on the market and are suitable for long term administration possess this property-sotalol and amiodarone. ${ }^{14-18}$ Previous studies suggest that both these drugs are effective in the management of refractory atrial fibrillation. However, the data must be interpreted with caution because of small sample sizes, short follow up, and the fact that most of the studies were not controlled or randomised.

This prospective, randomised, single blind trial was designed: first, to determine whether amiodarone and sotalol are superior to placebo for the long term maintenance of sinus rhythm in patients with recurrent, symptomatic atrial fibrillation; second, to examine the safety of the drugs when used in this setting; and third, to compare the two agents. Preliminary findings, including results from the patients in the amiodarone and sotalol groups during the first year of follow up, have already been published. ${ }^{19}$

\section{Methods}

One hundred and eighty six consecutive patients (97 men, 89 women; mean (SD) age 63 (10) years) who came to our emergency department or were admitted to our clinic with symptomatic chronic or paroxysmal atrial fibrillation were evaluated for enrolment in the study. Entry criteria included: age over 18 years; ECG documentation of atrial fibrillation; symptoms such as light headedness, palpitations, chest pain, and dyspnoea in association with atrial fibrillation; and successful chemical or electrical cardioversion to sinus rhythm in the patients with chronic atrial fibrillation. The exclusion criteria were: acute myocardial infarction less than seven days before entry to the trial; cardiogenic shock; transient atrial fibrillation related to an ongoing process such as pneumonia, pulmonary embolism, recent cardiac surgery, or uncontrolled congestive heart failure; unstable hepatic or renal function; echocardiographic ejection fraction $<40 \%$; hyperthyroidism; other major medical problems that would leave the patient with a life expectancy of less than one year; and 
previous exposure to either amiodarone or sotalol.

All patients gave their informed consent for inclusion in the study. The protocol was approved by the hospital's ethics committee.

\section{DEFINITIONS}

All patients were classified as having either chronic or paroxysmal atrial fibrillation as follows: paroxysmal atrial fibrillation was defined as recurrent, self terminating episodes lasting less than 48 hours, alternating with periods of sinus rhythm; patients with continuous atrial fibrillation, or in whom sinus rhythm had been present only briefly after previous pharmacological or electrical cardioversion, were classified as having chronic atrial fibrillation.

DRUG TREATMENT SCHEDULE

After restoration of sinus rhythm, eligible patients were randomly assigned in a single blinded fashion to receive either amiodarone, sotalol, or placebo. Before the start of treatment, all other antiarrhythmic drugs were discontinued for $\geqslant 5$ half lives. Treatment of underlying heart disease was optimised and kept constant in all patients throughout the study.

Patients randomised to amiodarone began with a dose of $15 \mathrm{mg} / \mathrm{kg} /$ day for seven days, followed by $10 \mathrm{mg} / \mathrm{kg} /$ day for another seven days orally, after which the drug dose was tapered to maintenance levels over 7-12 days. Thereafter, treatment was maintained at a dose of $200 \mathrm{mg} /$ day.

Patients randomised to sotalol began with $80 \mathrm{mg}$ twice daily. The dose was titrated up in $40-80 \mathrm{mg}$ increments every 48 to 72 hours until a maximum dose of $480 \mathrm{mg}$ daily was reached. The final maintenance dose of sotalol was adjusted downwards if adverse effects were noted by the patients or if the rate corrected QT interval was $\geqslant 0.5$ seconds.

DATA COLLECTION AND FOLLOW UP

Before patients were entered into the study, baseline demographic data were obtained, all relevant cardiac diagnoses and medical history noted, and physical examination, 24 hour ambulatory ECG, ophthalmological examination, pulmonary function tests, chest $x$ ray, thyroid function tests, and laboratory results (electrolytes, renal and liver function tests) carried out. Cross sectional echocardiography was used to determine left atrial size and the left ventricular ejection fraction. All echocardiographic recordings were reviewed by two experienced observers.

Patients in the amiodarone group remained in hospital during the initial loading phase (7-14 days) and were then examined every week until the 30th day. Patients receiving sotalol stayed in hospital throughout the dose titration phase and for 48 hours afterwards. Subsequently, patients were followed up in the first, second, fourth, and sixth months, and at three month intervals thereafter. If symptoms suggesting arrhythmia recurrence were noted and confirmed using a transtelephonic ECG transmitter system (Cardiocare, Medtronic,
New York, USA), or if side effects were observed, additional examinations were performed.

At each scheduled or unscheduled follow up visit, history, physical examination, routine laboratory tests, ECG, rhythm strip, and 24 hour ambulatory monitoring (when clinically indicated) were repeated. Chest $x$ rays were performed every six months; pulmonary function tests and ophthalmological examinations were repeated every year.

At one year, a preliminary analysis of two of the groups (sotalol and amiodarone) was carried out, which included those patients who had been enrolled up to that time. ${ }^{19}$

Serum concentrations of the drugs studied were not routinely measured and thus did not affect the dosage schedules. In the case of sotalol, the dose reported represents the highest tolerated dose during the long term maintenance phase. For amiodarone, the objective was to give the lowest dose that would maintain sinus rhythm without producing adverse side effects. Previous amiodarone studies suggest that there is only a rough correlation between dose and serum concentrations, and no correlation between the latter and efficacy or toxicity. ${ }^{162021}$

\section{STATISTICAL ANALYSIS}

Patients eligible for the study were allocated to treatment (sotalol, amiodarone, or placebo) using a computer generated random number algorithm. Descriptive statistics are summarised as mean (SD) or per cent. Baseline continuous variables were compared across the three groups by analysis of variance, $\chi^{2}$, or Fisher's exact tests, as appropriate. Adverse event-free Kaplan-Meier product limit estimate curves were constructed and compared across the three treatment groups using the log-rank test. The dependent variable in the primary analysis was time to adverse events, defined as either relapse to atrial fibrillation or intolerable side effects, whichever occurred first. A secondary analysis assessing the efficacy of the three drugs in keeping the patients atrial fibrillation free (regardless of side effects) was also carried out. In that analysis patients who remained in sinus rhythm but who experienced side effects were censored from the analysis on the date the drug was discontinued. The time at risk began at the start of drug administration.

Kaplan-Meier curves were also constructed and compared by log-rank test to provide a univariate assessment of the prognostic value of selected clinical risk factors, echo derived descriptors, and so on. Continuous covariates (age, left atrial size, time from initial diagnosis of atrial fibrillation) were split at their statistical median or at appropriate prespecified cut off points.

In all analyses the criterion for significance was $5 \%$.

\section{Results}

Of the 186 patients, 65 were assigned to amiodarone, 61 to sotalol, and 60 to placebo. The three groups were comparable in terms of sex, 
age, left atrial size, left ventricular ejection fraction, time from initial diagnosis of atrial fibrillation, number of previous trials, percentage of patients with underlying heart disease, and pattern of atrial fibrillation (table 1).

The time between the restoration of sinus rhythm and the start of administration of amiodarone, sotalol, or placebo did not differ between the groups, at 12 (8), 14 (9), and 14 (8) hours, respectively.

The mean (SD) maintenance dose for patients treated with sotalol was $320(20) \mathrm{mg} /$ day (titration phase, 4 (2) days), while all patients treated with amiodarone received $200 \mathrm{mg} /$ day.

RESPONSE TO TREATMENT

Thirty one of the 65 patients receiving amiodarone relapsed to atrial fibrillation after an average of six months, compared with 47 of the 61 patients given sotalol, after an average of eight months, and 53 of the 60 placebo patients, after an average of four months. Eleven patients on amiodarone (nine of whom were women) experienced significant side effects requiring withdrawal of the treatment while they were still in normal sinus rhythm (two during the loading phase); another four who relapsed in the same group also had side effects. The average time to the appearance of these side effects was 16 months (range 0-24 months). Three patients in the sotalol group developed significant side effects during the titration phase.

The average follow up time for the non-end point patients was 21.6 (2.3) months in the amiodarone group, 22 (2) months in the sotalol group, and 22.3 (1.8) months in the control group.

The primary analysis, counting adverse side effects as an end point, indicated that both amiodarone and sotalol were superior to placebo for the suppression of recurrent symptomatic atrial fibrillation ( $\mathrm{p}<0.001$ for both) Amiodarone was superior to sotalol in the long term $(\mathrm{p}<0.001)$. Its superiority, however, only became apparent after the six month time point, as a result of a higher initial relapse rate in the amiodarone group. The average monthly progression rates to atrial fibrillation or side effects were $4.9 \%, 8.3 \%$, and $14.7 \%$ for amiodarone, sotalol, and placebo, respectively.

Figure 1A shows the Kaplan-Meier product limit estimate curves for the primary analysis. At one month, $87 \%$ of the patients taking sotalol remained in sinus rhythm and free of side

Table 1 Clinical characteristics of the patients studied

\begin{tabular}{llll}
\hline & $\begin{array}{l}\text { Amiodarone } \\
(n=65)\end{array}$ & $\begin{array}{l}\text { Sotalol } \\
(n=61)\end{array}$ & $\begin{array}{l}\text { Placebo } \\
(n=60)\end{array}$ \\
\hline Sex (M/F) (n) & $34 / 31$ & $32 / 29$ & $31 / 29$ \\
Age (years) & $63.2(9.0)$ & $62.8(8.0)$ & $62.8(9.6)$ \\
LA size (mm) & $43(7)$ & $44(5)$ & $44(6)$ \\
LV ejection fraction (\%) & $54(12)$ & $52(13)$ & $55(12)$ \\
Number of previous drug trials & $2.0(1.2)$ & $2.1(1.0)$ & $1.9(0.8)$ \\
Months since AF diagnosis & $9(6)$ & $10(8)$ & $8(7)$ \\
Underlying heart disease (n) & $24(36.9 \%)$ & $21(34.4 \%)$ & $21(35 \%)$ \\
Pattern of AF (paroxysmal:chronic) (n) & $42: 23$ & $39: 22$ & $40: 20$ \\
\hline
\end{tabular}

Values are mean (SD) unless stated. No significant differences were found between the three groups.

AF, atrial fibrillation; LA, left atrial; LV, left ventricular.
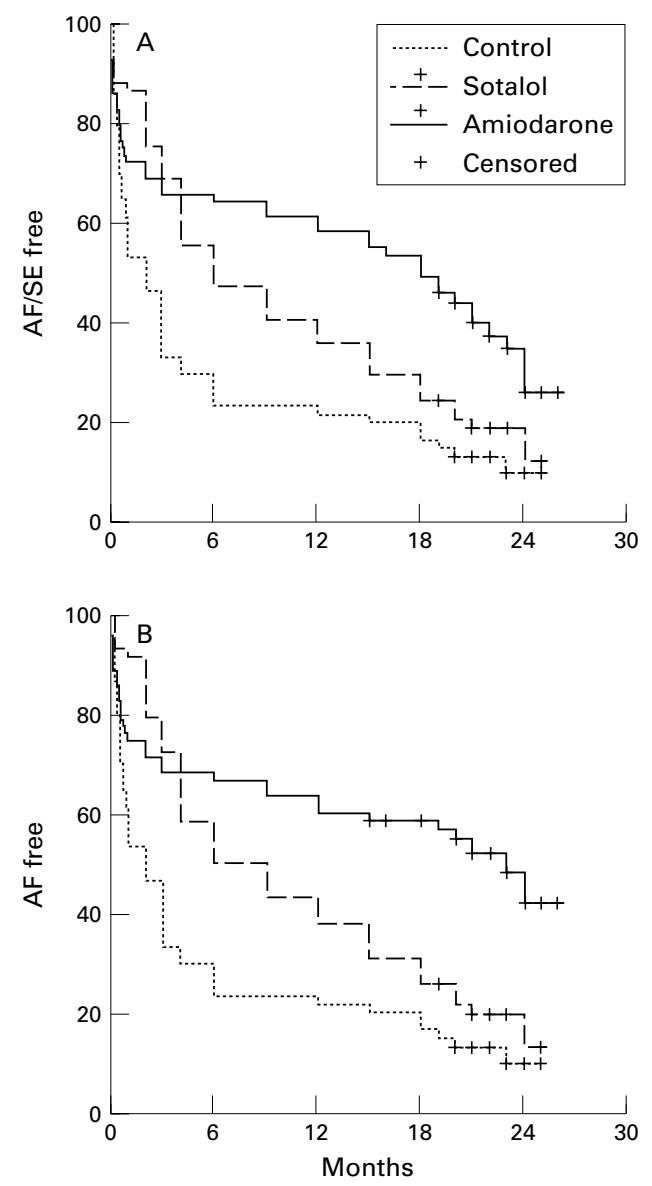

Figure 1 Kaplan-Meier curves for $(A)$ the primary analysis and $(B)$ the secondary analysis. Amiodarone and sotalol were superior to placebo. Amiodarone was better than sotalol, but its superiority only became evident after the six month point. When adverse effects are overlooked, amiodarone was seen to be much more effective than the other two agents. There was no difference in the pattern of censoring between the three groups. AF, atrial fibrillation; $S E$, side effects.

effects, compared with $72 \%$ of the amiodarone patients and $53 \%$ of the placebo group. From the sixth month the picture changed. After that time and for about one year, amiodarone patients showed a significantly reduced risk of atrial fibrillation compared with the sotalol group, who continued to progress to events at a fairly steady rate.

After 18 months the amiodarone patients appeared to progress at a faster rate to atrial fibrillation or side effects, so that by the second year the gap between amiodarone and the other two groups had narrowed. By the same time, the proportion of patients free of atrial fibrillation or side effects in the sotalol group had declined almost to that in the placebo group. The percentages of patients in sinus rhythm and free of side effects for amiodarone, sotalol, and placebo were $58.46 \%, 36.07 \%$, and $21.67 \%$, respectively, at one year, and $26.17 \%$, $12.61 \%$, and $10 \%$ at two years.

In the secondary analysis, when adverse effects were not counted as an end point, the picture changed (fig 1B). As expected, amiodarone was much more effective than sotalol or the placebo in maintaining normal sinus rhythm in the long term, and its superiority 
Table 2 Univariate assessment of risk factors

\begin{tabular}{|c|c|c|c|c|c|c|c|c|c|c|}
\hline \multirow[b]{2}{*}{ Risk factor } & \multicolumn{4}{|c|}{ Amiodarone } & \multicolumn{4}{|l|}{ Sotalol } & \multicolumn{2}{|l|}{ Placebo } \\
\hline & In $A F$ & $p$ Value & In $A F / S E$ & $p$ Value & In $A F$ & $p$ Value & In $A F / S E$ & $p$ Value & In $A F$ & $p$ Value \\
\hline \multicolumn{11}{|l|}{ Sex } \\
\hline Male & $21 / 34$ & & $23 / 34$ & & $26 / 32$ & & $28 / 32$ & & $28 / 31$ & \\
\hline Female & $10 / 31$ & 0.01 & $19 / 31$ & 0.14 & $21 / 29$ & 0.28 & $22 / 29$ & 0.24 & $25 / 29$ & 0.75 \\
\hline \multicolumn{11}{|l|}{ Age (years) } \\
\hline$\leqslant 65$ & $14 / 37$ & & $22 / 37$ & & $27 / 39$ & & $29 / 39$ & & $28 / 34$ & \\
\hline$>65$ & $17 / 28$ & 0.18 & $20 / 28$ & 0.57 & $20 / 22$ & 0.02 & $21 / 22$ & 0.04 & $25 / 26$ & 0.11 \\
\hline \multicolumn{11}{|l|}{ LA size $(\mathrm{mm})$} \\
\hline$\leqslant 44$ & $12 / 28$ & & $19 / 28$ & & $28 / 35$ & & $29 / 35$ & & $28 / 32$ & \\
\hline$>44$ & $19 / 37$ & 0.65 & $23 / 37$ & 0.77 & $19 / 26$ & 0.97 & $21 / 26$ & 0.85 & $25 / 28$ & 0.86 \\
\hline \multicolumn{11}{|c|}{$\begin{array}{l}\text { Months since } \mathrm{AF} \\
\text { diagnosis }\end{array}$} \\
\hline$\leqslant 6$ & $12 / 27$ & & $20 / 27$ & & $16 / 24$ & & $17 / 24$ & & $17 / 22$ & \\
\hline$>6$ & $19 / 38$ & 0.11 & $22 / 38$ & 0.60 & $31 / 37$ & 0.31 & $33 / 37$ & 0.25 & $36 / 38$ & 0.15 \\
\hline \multicolumn{11}{|c|}{$\begin{array}{l}\text { Underlying heart } \\
\text { disease }\end{array}$} \\
\hline Yes & $12 / 24$ & & $15 / 24$ & & $16 / 21$ & & $17 / 21$ & & $19 / 21$ & \\
\hline No & $19 / 41$ & 0.12 & $27 / 41$ & 0.81 & $31 / 40$ & 0.21 & $33 / 40$ & 0.35 & $34 / 39$ & 0.46 \\
\hline \multicolumn{11}{|l|}{ AF pattern } \\
\hline Paroxysmal & $22 / 42$ & & $30 / 42$ & & $31 / 39$ & & $33 / 39$ & & $36 / 40$ & \\
\hline Chronic & $9 / 23$ & 0.65 & $12 / 23$ & 0.30 & $16 / 22$ & 0.53 & $17 / 22$ & 0.52 & $17 / 20$ & 0.48 \\
\hline
\end{tabular}

For amiodarone and sotalol, findings from both the primary (in AF/SE) and the secondary analysis (in AF) are shown.

$\mathrm{AF}$, atrial fibrillation; LA, left atrial; SE, side effects.

persisted after the 18 month mark, when amiodarone patients experienced frequent side effects. The 12 month atrial fibrillation free percentage was now $60.3 \%$ for amiodarone, compared with $37.9 \%$ for sotalol and $21.67 \%$ for placebo, while the corresponding two year figures were $42.6 \%, 13.3 \%$, and $10 \%$.

All but one of the 11 patients who were still in sinus rhythm but were censored from the analysis because of side effects progressed to atrial fibrillation, on average four months (range 3-6 months) after treatment was discontinued. The single patient who remained in sinus rhythm was a 65 year old woman.

PREDICTORS FOR RECURRENCE OF ATRIAL FIBRILLATION

As the proportion of patients remaining in sinus rhythm showed significant differences between the three groups, the predictors for recurrence of atrial fibrillation were examined separately (table 2).

In the amiodarone group univariate analysis showed that none of the factors we considered (sex, age, left atrial size, time from initial diagnosis, underlying heart disease, or pattern of atrial fibrillation) significantly affected the progression to atrial fibrillation. However, when adverse side effects were overlooked, sex became a significant factor. Female patients had a much better chance of remaining in normal sinus rhythm, without side effects, for a longer period of time (one year rates: men $48.5 \%$, women $76.7 \%$; two year rates: men $31.5 \%$, women $56.6 \%$ ) (fig 2). This was because nine of the 11 patients in this group who experienced side effects were women.

In the sotalol group, univariate assessment of the factors that affect progression to atrial fibrillation showed that age was the only significant predictor: patients less than 65 years old had a much better chance of remaining in normal sinus rhythm for a longer time.

No factor appeared to affect the progression to atrial fibrillation in the placebo group.

Amiodarone was superior to sotalol and placebo, regardless of left atrial size, sex, age, time from initial diagnosis, underlying heart disease, or pattern of atrial fibrillation.

ADDITIONAL ANALYSIS

Because of the difference in the pharmacokinetics of amiodarone and sotalol, we performed an additional analysis, discounting recurrences during the loading or titration
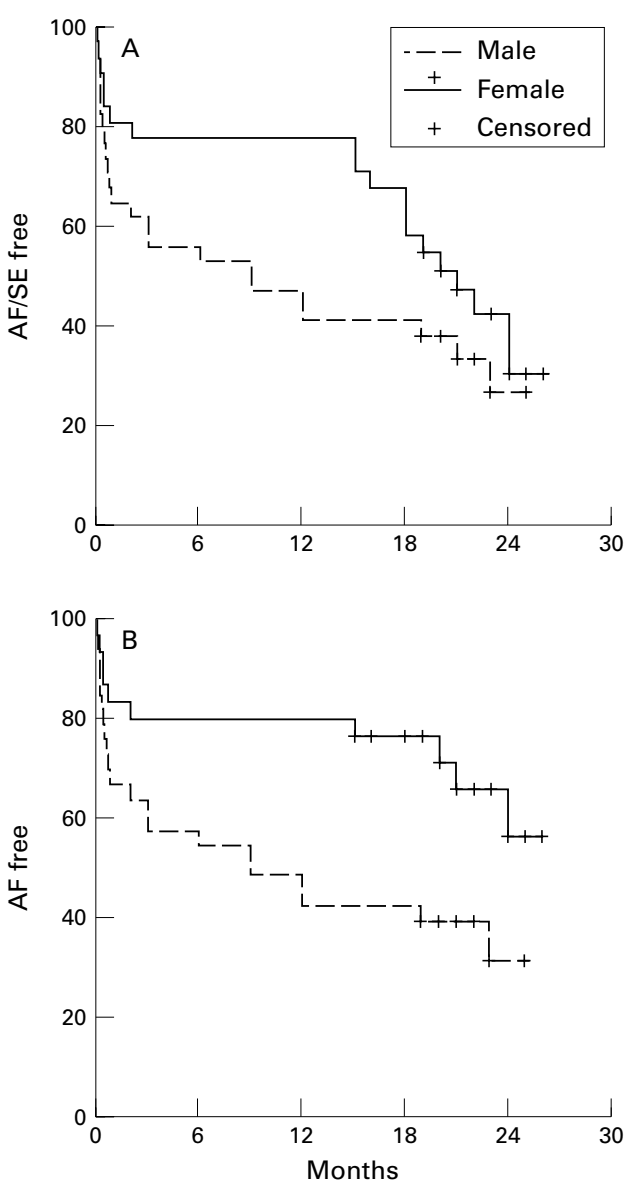

Figure 2 Kaplan-Meier curves for $(A)$ the primary analysis, and (B) the secondary analysis, grouped by sex in the amiodarone group. Female patients had a much better chance of remaining in sinus rhythm for a longer period, but also experienced more side effects than men. 


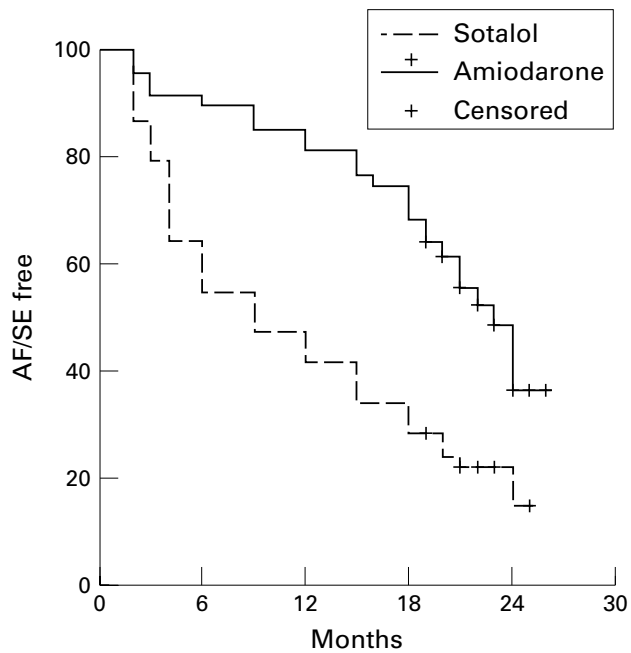

Figure 3 Progression to atrial fibrillation and side effects, with the first month's relapses censored. Amiodarone is now superior to sotalol throughout the period of observation.

phase. In other words, we censored the relapses occurring in both groups during the one month period required to obtain the full effect of amiodarone, the slower acting of the two drugs.

On this basis, amiodarone was superior $(\mathrm{p}<0.001)$ to sotalol in keeping patients free of atrial fibrillation and side effects throughout the period of observation (fig 3). The percentages of patients in sinus rhythm at six, 12 , and 24 months were $89.36 \%, 80.85 \%$, and $36.2 \%$ for amiodarone, and $54.72 \%, 41.51 \%$, and $14.51 \%$ for sotalol, respectively.

\section{ADVERSE EFFECTS}

Neither proarrhythmia nor sudden death occurred in any patient during the study.

In the amiodarone group 15 patients experienced significant adverse effects. Two of them (one man, one woman) developed symptomatic bradycardia during the loading phase. These two patients were relatively bradycardic even before the administration of the drug, which made the condition worse and led to their exclusion from the study. Eight patients (all women) developed hypothyroidism, four (three men, one woman) developed hyperthyroidism, while one patient (a man) developed ataxia. In all cases amiodarone was discontinued and appropriate treatment was given. Minor side effects, predominantly gastrointestinal discomfort, nausea, sun sensitivity, and ophthalmic problems, occurred in $9 \%$ of the patients. In all cases, these problems disappeared spontaneously or were eliminated by dose reduction, splitting the dose, or avoiding exposure to sunlight.

In the sotalol group two patients developed symptomatic bradycardia and one severe dizziness. All of these developed the side effects at the lowest dose during the titration phase and treatment was discontinued.

None of the patients in the placebo group developed side effects.

\section{Discussion}

Our study showed that treatment with amiodarone and sotalol is more effective than no treatment for the long term maintenance of normal sinus rhythm in patients with recurrent symptomatic atrial fibrillation. Our findings also extend and complete those of a previous preliminary report, ${ }^{19}$ showing that amiodarone is the more effective of the two drugs for this purpose, but causes more side effects. Amiodarone and sotalol are two antiarrhythmic agents which belong to class III, according to the Singh-Vaughan Williams classification. However, neither drug is a selective class III agent. Amiodarone also has class I, calcium channel blocking effects and an anti-adrenergic action, while sotalol is also a non-selective $\beta$ blocker. These extra properties might influence not only the efficacy but also the safety of the two drugs when used for the treatment of atrial fibrillation.

\section{EFFICACY OF AMIODARONE}

This is the first randomised, single blinded study to show that treatment with amiodarone is more effective than no treatment in maintaining normal sinus rhythm in patients with recurrent symptomatic atrial fibrillation. According to our findings, although the percentage of patients receiving amiodarone who remained in sinus rhythm and free of side effects declined with time, after two years this percentage was more than double that for placebo. It should be noted that, even during the loading phase when the full action of amiodarone had not been attained, there were significantly fewer relapses than in patients receiving placebo.

A factor which reduces the long term benefit of amiodarone is the appearance of various side effects. In our study of two years' treatment, when arrhythmia recurrence and adverse effects were both considered as treatment failure, the overall efficacy of amiodarone was low (26.17\% of patients in sinus rhythm and event free) while it was much higher $(42.6 \%$ in sinus rhythm) when adverse effects were overlooked. The latter observation could suggest a basis for the view that side effects should as far as possible be treated and that amiodarone should not be discontinued when maintenance of sinus rhythm is judged to be essential. ${ }^{12} 2223$

Regarding the success rate, our results are comparable with those of most non-placebo controlled studies. ${ }^{2-589111524}$ Only the study of Chun and colleagues found much higher percentages. ${ }^{11}$ However, in that study the patients who relapsed to atrial fibrillation during the first month of treatment were censored and, as our additional analysis showed, this would have had an inflating effect on the success rates.

Our findings suggest that the only factor that affects the maintenance of sinus rhythm with amiodarone is the sex of the patient. Female patients have a much better chance of remaining in normal sinus rhythm for a longer period, provided that they can tolerate amiodarone treatment. This finding was unexpected and is difficult to explain. Only one earlier study has reported that sex plays a role and in that case the findings were the opposite to ours. ${ }^{25}$ However, our findings regarding predictors should be viewed with caution as in our control group 
no predictive factors were apparent, in contrast to other reports. This was probably because in our present study the time from the initial diagnosis of atrial fibrillation was short, while most patients had normal left atrial dimensions and heart size.

EFFICACY OF SOTALOL

Our study also proves that sotalol is more effective than no treatment in maintaining normal sinus rhythm in patients with recurrent symptomatic atrial fibrillation.

According to our findings, after one year sotalol treated patients have a $70 \%$ greater chance than untreated patients of remaining in sinus rhythm and free of side effects $(36 \% v$ $21 \%$ ). However, after two years almost all our patients had reverted to atrial fibrillation. The latter observation is new, as our study lasted longer than any previous one. The fact that our results on the effectiveness of sotalol at the one year follow up were comparable with those of previous placebo and non-placebo controlled studies with the same length of follow up tends to confirm the validity of our long term findings. . $^{2-5} 8-101317$

With regard to factors affecting the efficacy of sotalol, our findings appear to indicate that only age $(<65$ years) plays an important role. However, the same caveat applies as in the case of amiodarone. Previous studies have reported that factors such as atrial size, duration of atrial fibrillation, and the number of episodes in paroxysmal atrial fibrillation affect the progression to atrial fibrillation during treatment with sotalol. $^{2-5} 1013$

SAFETY

Our findings on the safety of amiodarone are in agreement with those of previous studies. ${ }^{311-1318} 23$ Even though we used a low maintenance dose, we found that amiodarone caused various non-cardiac side effects, the incidence of which increased over time. This may be because many side effects are related to the total dose given, or to the total dose given over time - that is, to the amount of drug that has accumulated.

However, the observation that side effects are more common in women is new. As the most frequent side effect in our population was hypothyroidism, and as it is well known that women who have pre-existing thyroid antibodies are at increased risk of amiodarone induced hypothyroidism (13.5 times that of men without such antibodies), ${ }^{22}{ }^{23}$ it is possible that our female patient population contained a high proportion of patients with such antibodies. Unfortunately, we did not test for thyroid antibodies before amiodarone administration and so this hypothesis could not be verified.

With regard to sotalol, our study shows that it is relatively safe. A small number of patients showed side effects early on, during the titration phase. Previous studies, however, have reported a significant proarrhythmic effect, especially in patients with underlying heart disease. $^{3-5}$ 8-10 13 The fact that patients with severe heart disease, such as myocardial infarction, cardiogenic shock, an ejection fraction of less than $40 \%$, and so on, were excluded from our study obviously had a significant effect on the findings concerning the safety of sotalol.

\section{COMPARISON BETWEEN AMIODARONE AND}

SOTALOL

Previous non-randomised studies suggest that amiodarone is more effective than sotalol in the treatment of atrial fibrillation and that it entails a lower risk of proarrhythmia, though causing more non-cardiac side effects. ${ }^{3-5}$ 8-13 However, until now there has been no study that has compared the two agents directly (apart from our own preliminary findings which have already been published ${ }^{19}$ ). In this study we compared for the first time the effectiveness and safety of the two drugs for the maintenance of sinus rhythm in patients with refractory atrial fibrillation, and our results confirm the above hypothesis.

The superiority of sotalol during the first months of our study was, as shown by the additional analysis, caused by the delayed action of amiodarone. However, we cannot exclude the possibility that the loading dose we used was inadequate. Whether a better loading regimen would reduce the difference in the early effectiveness of the two drugs remains to be investigated in future studies.

The above results are not surprising. As already mentioned, although the two agents belong to the same class (III), they both display other pharmacological properties that differentiate them. ${ }^{13-15}$ One fundamental difference in their actions is that amiodarone does not exert frequency dependent effects on repolarisation, in contrast to sotalol which is characterised by a progressive loss of effect at higher stimulation rates, otherwise known as "reverse use dependence". This difference between sotalol and amiodarone could explain the different antiarrhythmic and proarrhythmic effects of the two compounds and, by extension, the better efficacy of amiodarone.

METHODOLOGICAL CONSIDERATIONS

Any study designed to evaluate the efficacy of treatment for atrial fibrillation must address several methodological problems. Critically important among these is that of documenting cardiac rhythm before and after treatment. In the present trial, recurrences were determined by relapse of symptoms or by assessment of the ECG at certain time points. Therefore, recurrences of arrhythmia that were asymptomatic because of a reduced ventricular response to atrial fibrillation caused by the treatment may have gone undetected. Although the modification of arrhythmia recurrence from symptomatic to asymptomatic could be regarded as a beneficial therapeutic response, this remains a limitation of the study. However, previous studies have shown that few patients (less than $10 \%$ ) have asymptomatic recurrences of atrial fibrillation, so the probable effect on our results is relatively small. ${ }^{8}{ }^{26}$ Furthermore, this limitation has no bearing on the superiority of amiodarone over sotalol: rather, it would tend to lead to underestimation of the difference, as the percentage of undetected relapses might be 
expected to be higher in patients taking sotalol, which has a greater negative chronotropic effect than amiodarone.

Another methodological problem faced by any study of drug efficacy in the suppression of atrial fibrillation is whether to use as an end point the number of episodes of atrial fibrillation during the period of observation or the time to first relapse. Although the number and the severity of atrial fibrillation relapses are fundamental in the evaluation of the efficacy of an agent, in our study only the time to first relapse was studied. This was because reliable pretrial relapse rates were not available for all patients and are not applicable to those with chronic atrial fibrillation.

Finally, it should be noted that patients with an ejection fraction of less than $40 \%$ were excluded from our study. We decided to do this because of the high probability of such patients developing side effects from sotalol. However, as a result we do not know whether our results - particularly for amiodarone - apply to patients with poor left ventricular function. Given that amiodarone is well tolerated by these patients it would be interesting in future studies to investigate the effectiveness of the drug in that context.

\section{STUDY IMPLICATIONS}

In this study we used low doses of amiodarone for the maintenance of sinus rhythm and compared the results with those from the largest possible doses of sotalol and with placebo. This decision was designed to avoid most of the side effects of amiodarone, which are known to be dose dependent.

Our results showed that both amiodarone and sotalol can be used for the maintenance of normal sinus rhythm in patients with recurrent symptomatic atrial fibrillation, but that low dose amiodarone is more effective than high doses of sotalol. Unfortunately, in spite of the low amiodarone dosage we were unable to avoid side effects after long term treatment, perhaps because many of these effects are related to the total dose given or to the total dose given over time. However, in cases where the maintenance of sinus rhythm is considered to be essential, amiodarone treatment should be considered, in combination with the treatment of any side effects.

We would like to thank Dr Stavros Chrysostomakis and Mr We would like to thank Dr Stavros Chrysostomakis and $\mathrm{Mr}$
Philip Lees for their assistance in the preparation of the text and illustrations, and Mr Gregory Chlouverakis for the statistical analyses.

1 Coplen SE, Antman EM, Berlin JA, et al. Efficacy and safety of quinidine therapy for maintenance of sinus rhythm after cardioversion: a meta-analysis of randomized control trials. Circulation 1990;82:1106-16.
2 Reimold SC. Clinical challenge I: control of recurrent symptomatic atrial fibrillation. Eur Heart f 1996;17(suppl C):35-40

3 Nattel S. Newer developments in the management of atrial fibrillation. Am Heart f 1995;130:1094-106.

4 Waldo AL, Prystowsky EN. Drug treatment of atrial fibrillation in the managed care. Am f Cardiol 1998;81(5A):2329C.

5 Prystowsky EN, Benson DW, Fuster V, et al. Management of patients with atrial fibrillation. A statement for healthcare professionals, from the subcommittee on electrocardiography and electrophysiology, American Heart Association. Circulation 1996;93:1262-77.

6 Porterfield JG, Porterfield LM. Therapeutic efficacy and safety of oral propafenone for atrial fibrillation. Am 7 Cardiol 1989;63:114-16.

7 Chimienti M, Cullen MT, Casadei G. Safety of flecainide versus propafenone for the long-term management of symptomatic paroxysmal supraventricular tachyarrhythmias. Report from the Flecainide and Propafenone Italian Study (FAPIS) Group. Eur Heart f 1995;16:1943-51.

8 Antman EM, Beamer AD, Cantillon C, et al. Therapy of refractory symptomatic atrial fibrillation and atrial flutter. A staged care approach with new antiarrhythmic drugs. $\mathcal{F}$ Am Coll Cardiol 1990;15:698-707.

9 Edvardsson N. Comparison of class I and class III action in atrial fibrillation. Eur Heart f 1993;14(suppl H):62-6.

10 Juul-Moller S, Edvardsson N, Rehnqvist-Ahlberg N. Sotalol versus quinidine for the maintenance of sinus rhythm after direct current conversion of atrial fibrillation. Circulation 1990;82:1932-9.

11 Chun SH, Sager PT, Stevenson WG, et al. Long-term efficacy of amiodarone for the maintenance of normal sinus rhythm in patients with refractory atrial fibrillation or flutter. Am 7 Cardiol 1995;76:47-50.

12 Middlekauff HR, Wiener I, Stevenson WG. Low-dose amiodarone for atrial fibrillation. Am $\mathcal{F}$ Cardiol 1993;72:75$81 \mathrm{~F}$.

13 Singh BN, Ahmed R. Class III antiarrhythmic drugs. Curr Opin Cardiol 1994;9:12-22.

14 Wang J, Bourne GW, Wang Z, et al. Comparative mechanisms of antiarrhythmic drug action in experimental fibrillation. Importance of use-dependent effects on refractoriness. Circulation 1993;88:1030-44.

15 Singh BN. Controlling cardiac arrhythmias by lengthening repolarization: historical overview. Am f Cardiol 1993;72: $18-24 \mathrm{~F}$.

16 Hohnloser SH, Meinertz T, Dammbacher $\mathrm{T}$, et al. Electrocardiographic and antiarrhythmic effects of intravenous amiodarone: results of a prospective, placebocontrolled study. Am Heart f 1991;121:89-95.

17 Benditt DG, Williams JH, Jin J, et al. Maintenance of sinus rhythm with oral d,1-sotalol therapy in patients with symptomatic atrial fibrillation and/or atrial flutter. Am $\mathcal{F}$ Cardiol 1999;84:270-7.

18 Southworth MR, Zarembski D, Viana M, et al. Comparison of sotalol versus quinidine for maintenance of normal sinus rhythm in patients with chronic atrial fibrillation. $A m \mathcal{F}$ Cardiol 1999;83:1629-32.

19 Kochiadakis GE, Igoumenidis NE, Marketou ME, et al. Low dose amiodarone versus sotalol for suppression of recurrent symptomatic atrial fibrillation. Am $\mathcal{f}$ Cardiol 1998;81:995-8.

20 Scheinman MM, Levine JH, Cannom DS, et al. A dose-ranging study of intravenous amiodarone in patients with life-threatening ventricular tachyarrhythmias. The Intravenous Amiodarone Multicenter Investigators Group. Circulation 1995;92:3264-72

21 Rotmensch HH, Belhassen B, Swanson BN, et al. Steady-state serum amiodarone concentrations: relationships with antiarrhythmic efficacy and toxicity. Ann Intern Med 1984;101: 462-9.

22 Jafari-Fesharaki $M$, Scheinman MM. Adverse effects of amiodarone. PACE 1998;21:108-20.

23 Harjai KJ, Licata AA. Effects of amiodarone on thyroid function. Ann Intern Med 1997;126:63-73.

24 Gold RL, Haffajee CI, Charos G, et al. Amiodarone for refractory atrial fibrillation. Am f Cardiol 1986;57:124-7.

25 Suttorp MJ, Kingma JH, Koomen EM, et al. Recurrence of paroxysmal atrial fibrillation or flutter after successful cardioversion in patients with normal left ventricular function. Am f Cardiol 1993;71:710-13.

26 Antman EM, Ludmer PL, McGowan N, et al. Transtelephonic electrocardiographic transmission for management of cardiac arrhythmias. Am $\mathcal{F}$ Cardiol 1986;58:1021-4. 